\title{
HONGOS ENDÓFITOS SEPTADOS, PERO NO LOS MICORRÍZICOS ARBUSCULARES AFECTAN EL CRECIMIENTO TEMPRANO DE QUERCUS HINTONII, UN ENCINO ENDÉMICO DEL ESTADO DE MÉXICO
}

\section{SEPTATE ENDOPHYTIC FUNGI, BUT NOT ARBUSCULAR MYCORRHIZAL FUNGI AFFECT THE EARLY GROWTH OF QUERCUS HINTONII, AN ENDEMIC OAK FROM MEXICO STATE}

\author{
@irma Reyes-Jaramillo, @Noé Manuel Montaño*, @Eber Silva-González \\ Departamento de Biología, División de Ciencias Biológicas y de la Salud, Universidad Autónoma Metropolitana-Iztapalapa, \\ México.
}

*Autor de correspondencia: nmma@xanum.uam.mx

\begin{abstract}
Resumen
Antecedentes: Los encinos se asocian con hongos micorrízicos y endofíticos septados, pero se desconoce cómo estos hongos impactan su desarrollo.

Pregunta: ¿Cómo la interacción entre hongos micorrízicos arbusculares (HMA) y endófitos septados (HES) afecta el desarrollo de $Q$. hintonii?

Especies de estudio: Quercus hintonii

Sitio de estudio y fechas: Tejupilco, Estado de México, 2015

Método: Dos experimentos con plántulas fueron diseñados: I) Sphagnum (Sph) con y sin inóculo micorrízico, y II) Suelo forestal natural (SFN) y Sphagnum, ambos sin (SFN/Sph) y con inóculo micorrízico $(\mathrm{SFN}+\mathrm{I} / \mathrm{Sph}+\mathrm{I})$. Se evaluó el crecimiento de las plántulas y la colonización fúngica radical a 4, 6 y 7 meses (Exp. I), y a los 4 meses (Exp. II).

Resultados: Las raíces de $Q$. hintonii no fueron colonizadas por HMA, sino por HES, que desarrollaron células moniliformes (32\%), micelio septado $(51 \%)$ y otras estructuras (7\%). Las plántulas de seis y siete meses tuvieron menor colonización (12-14 \%). Las plántulas de cuatro meses y con mayor colonización por HES tuvieron más biomasa total, número de hojas, peso seco de hojas y raíces, y tallos más gruesos. En el experimento II, las plántulas en SFN tuvieron mayor longitud de raíz y biomasa seca que en SFN+I, Sph+I y Sph. El micelio septado y microesclerocios de HES fueron abundantes en plántulas de SFN y SFN+I.

Conclusiones: Los HES nativos colonizando las raíces de $Q$. hintonii favorecen el crecimiento de las plántulas al inicio de su desarrollo; mientras que los HMA no interaccionaron con este encino.
\end{abstract}

Palabras clave: Encinos, hongos endofíticos, inóculo microbiano, micorriza, microesclerocios.

\begin{abstract}
Background: The oaks are associated with mycorrhizal and septate-endophytic fungi; however, we unknown how these fungi impact its development.

Question: How does the interaction between arbuscular mycorrhizal fungi (AMF) and septate endophytic fungi (SEF) affect the development of $Q$. hintonii?

Species study: Quercus hintonii

Study site and years of study: Tejupilco region, State of Mexico, 2015

Methods: Two experiments with oak seedlings were designed: I) Sphagnum (Sph) with and without mycorrhizal inoculum, and II) Natural forest soil (NFS) and Sphagnum without (NFS/Sph) and with mycorrhizal inoculum (NFS+I/Sph+I). Seedlings growth and its roots fungal colonization were evaluated at 4, 6 and 7 old months (Exp. I), and at 4 old months (Exp. II).

Results: The $Q$. hintonii roots were not colonized by AMF, but if by SEF, which developed moniliform cells (32\%), septate mycelium (51\%) and other structures (7\%). Seedlings of six and seven old months had lower colonization by SEF (12-14 \%). Oak inoculated seedlings of 4old months and with higher colonization by SEF, had greater total biomass, number of leaves, dry weight of leaves and roots, and thicker stems. In experiment II, $Q$. hintonii seedlings grown in NFS had greater root length and total biomass than those grown in NFS+I, Sph+I, and Sph. Septate mycelium and microsclerotia of SEF were abundant in NFS and NFS+I.

Conclusions: The colonization by native SEF in $Q$. hintonii roots favored early development of the seedlings, while the AMF did not interact with this oak species.
\end{abstract}

Keywords: Fungal endophytes, microbial inoculum, microsclerotia, mycorrhiza, oaks. 
Los encinos son árboles y en menor cantidad arbustos pertenecientes al género Quercus (Fagaceae), junto con los pinos son elementos característicos con la mayor biomasa en los bosques templados del mundo (Cavender-Bares 2018), estos bosques se consideran el segundo tipo de vegetación de importancia en México por su amplia distribución (Rzedowski 2006) y por albergar cerca del $40 \%$ de las especies de Quercus (161 spp.) hasta ahora reportadas; por lo que México, por su alta riqueza específica y elevado endemismo (Valencia-A 2004), se considera el segundo centro de diversificación del género en el mundo (Romero-Rangel et al. 2015).

Los encinos ejercen una fuerte influencia sobre la composición fúngica del suelo (Toju et al. 2013, Kwaśna et al. 2016), favoreciendo principalmente a los phyla Ascomycota y Basidiomycota, como se demostró para $Q$. castanea Mill. y $Q$. deserticola Trel. en un bosque templado de Michoacán en México (Chávez-Vergara et al. 2016). Otros estudios indican que las raíces finas de las especies de Quercus se asocian principalmente con hongos ascomicetos y basidiomicetos formadores de ectomicorrizas (Southworth 2013); sin embargo, éstas también pueden asociarse con hongos micorrízicos arbusculares (HMA) (Toju et al. 2013) y favorecer incluso la presencia de especies de HMA probablemente exclusivas del bosque de encino (Daniels \&Trappe 1979, González-Cortés et al. 2012, Varela-Fregoso et al. 2017). Aunque se menciona que en bosques templados la ectomicorriza predomina sobre la micorriza arbuscular (Read \& Pérez-Moreno 2003), ambas pueden colonizar simultáneamente las raíces de varias especies de encino, como se ha reportado en Quercus agrifolia Née y $Q$. garryana Douglas ex Hook. (Valentine et al. 2002), así como en Q. rubra L. y Q. palustris Münchh. (Dickie et al. 2001, Bainard et al. 2011). Asimismo, en $Q$. rugosa Née se ha observado, por ejemplo, que la micorriza arbuscular ocurre en los primeros meses de edad de este encino, pero posteriormente es desplazada por la ectomicorriza (Olivera-Morales et al. 2011).

Además de los HMA y ectomicorrízicos, también se reporta la presencia de otros hongos en las raíces de los encinos, tal es el caso de los hongos endófitos septados obscuros (HESO), los cuales son hongos anamórficos que colonizan intra- e intercelularmente las raíces, y que pueden cohabitar con los HMA o ser considerados como micorrízicos bajo algunas condiciones (Jumpponen 2001, Heredia-Acuña et al. 2014). En un bosque templado de Japón, dominado por Q. serrata Murray, Toju et al. (2013) registraron que el $75 \%$ de las raíces de esta especie estaban colonizadas tanto por hongos ectomicorrízicos como por HESO, y el $1.9 \%$ por HMA, sugiriendo una amplia coocurrencia de hongos en la raíz de $Q$. serrata, en donde los ascomicetos endofíticos fueron más comunes que los basidiomicetos ectomicorrízicos. No obstante, los HESO podrían no ser los únicos endofíticos en las raíces, ya que otros hongos endófitos septados (HES), con hifas hialinas, también han sido registrados en las raíces de varias especies de plantas (Porras-Alfaro \& Bayman 2011).

Las asociaciones ecto y endomicorrízicas favorecen el crecimiento y nutrición de las plantas, así como la estructura del suelo (Read \& Pérez-Moreno 2003); en particular en los bosques templados estas asociaciones son esenciales, debido a que permiten la captación de fósforo en donde la acidez edáfica limita la disponibilidad de este nutrimento (Romero-Rangel et al. 2015). Por otra parte, algunos estudios muestran que los HESO y HES pueden también promover el crecimiento de la planta hospedera, inhibir patógenos y mejorar la tolerancia al estrés biótico y abiótico (Arnold \& Herre 2003, Herrera et al. 2007, Rodriguez \& Redman 2008, Porras-Alfaro \& Bayman 2011, Shankar Naik 2019, Yan et al. 2019). Se ha reportado que plantas inoculadas con HESO incrementaron su biomasa de brotes y raíces, así como la concentración de $\mathrm{N}$ y $\mathrm{P}$ en su tejido, sugiriendo que estos hongos pueden actuar como sustitutos de los micorrízicos en hábitats polares y alpinos (Newsham et al. 2009); sin embargo, también se han señalado efectos negativos y neutrales, e incluso que éstos pueden reemplazar a los HMA bajo algunas condiciones (HerediaAcuña et al. 2014). Asimismo, los HES colonizando las raíces también confieren resistencia al estrés biótico y abiótico e influencian la adecuación de sus hospederos al afectar el reciclamiento de la materia orgánica y promover la captura de nutrimentos (Shankar Naik 2019, $\underline{\text { Yan et al. }}$ 2019).

La amplia distribución de los HESO y HES asociados con las raíces finas de árboles y arbustos prevaleciendo en ambientes con alto estrés ambiental en los biomas de todas las latitudes (Rodriguez et al. 2009, Porras-Alfaro \& Bayman 2011), y el hecho de que las raíces de los encinos estén colonizadas por una amplia variedad de hongos, con diversas funciones y múltiples efectos o beneficios en éstos hospederos, sugiere que las asociaciones micorrízicas y endofíticas podrían ser esenciales en la sobrevivencia y crecimiento de los encinos (Southworth 2013). No obstante, aún son limitados los estudios acerca de la interacción entre los HMA y los hongos endofíticos septados, así como de su efecto en el desarrollo de las plántulas de varios géneros que habitan en bosques templados, entre ellos Quercus (Heredia-Acuña et al. 2014). Por lo tanto, el objetivo de este estudio fue evaluar el efecto de los HMA y hongos endofíticos septados en el desarrollo de plántulas de $Q$. hintonii E.F. Warb., un encino endémico de los bosques templados en la región de Tejupilco, en el Estado de México, México. 


\section{Materiales y métodos}

Área de estudio. Esta investigación se realizó con material recolectado en Rincón de Ugarte, Municipio Tejupilco de Hidalgo, Estado de México, México, ubicado entre las coordenadas $18^{\circ} 56^{\prime} 27^{\prime \prime} \mathrm{N}$ y $100^{\circ} 09^{\prime} 20.9 \mathrm{O}$, a una altitud de $1,500 \mathrm{~m}$ snm. (Figura 1A). El tipo de vegetación corresponde a un bosque templado de encino caducifolio, en el cual $Q$. hintonii es una especie endémica dominante, cuya distribución ocurre principalmente en las provincias biogeográficas mexicanas Sierra Madre del Sur y Cuenca del Balsas. El clima es semicálido subhúmedo con lluvias en verano (INEGI 2009), con una temperatura media anual de $23{ }^{\circ} \mathrm{C}$ y una precipitación promedio anual de $1,300 \mathrm{~mm}$. Las lluvias más abundantes ocurren de junio a septiembre, con una marcada estación seca. El suelo dominante es Cambisol dístrico con textura media, $6.8 \%$ de materia orgánica, $0.5 \%$ de nitrógeno y 2 ppm de fósforo asimilable, con muy baja capacidad de intercambio catiónico $\left(14 \mathrm{cmol}_{(+)} \mathrm{kg}^{-1}\right)$ y un $\mathrm{pH}$ (4.6) fuertemente ácido (ReyesJaramillo 2006).

Recolecta de material biológico y experimento de inoculación. Se recolectaron bellotas viables de $Q$. hintonii en el mes de agosto de 2012, las cuales fueron almacenadas en contendores de plástico (Díaz-Pontones \& ReyesJaramillo 2009) y seleccionadas por medio del método de flotación (Bonner \& Vozzo 1987). Las bellotas seleccionadas fueron sembradas en tres charolas con tubetes de $20 \mathrm{~g}$ de capacidad, usando como sustrato musgo comercial (Sphagnum -Sph-). Se utilizaron 50 bellotas de $Q$. hintonii por charola (Figura 1B, C), las bellotas se escarificaron con una lija y se esterilizaron en superficie con hipoclorito de sodio en una relación 1:1 m/v. Además, se recolectó suelo forestal natural (SFN) asociado al encino, de los primeros $15 \mathrm{~cm}$ de profundidad; el cual fue colocado en bolsas negras etiquetadas, que fueron trasladadas al laboratorio y almacenadas bajo refrigeración $\left(4{ }^{\circ} \mathrm{C}\right)$ hasta usarse en los experimentos.

Se realizaron dos experimentos que consistieron en diferentes tratamientos. En el experimento I, los tratamientos fueron: i) Sphagnum sin inóculo micorrízico arbuscular (Sph), como control) y ii) Sphagnum con inóculo micorrízico arbuscular $(\mathrm{Sph}+\mathrm{I})$, con 25 réplicas cada uno. En el experimento II los tratamientos fueron: i) Sphagnum (Sph) esterilizado, como control), ii) Suelo forestal natural (SFN), como control), iii) SFN más inóculo micorrízico arbuscular $(\mathrm{SFN}+\mathrm{I})$ y iv) Sphagnum más inóculo
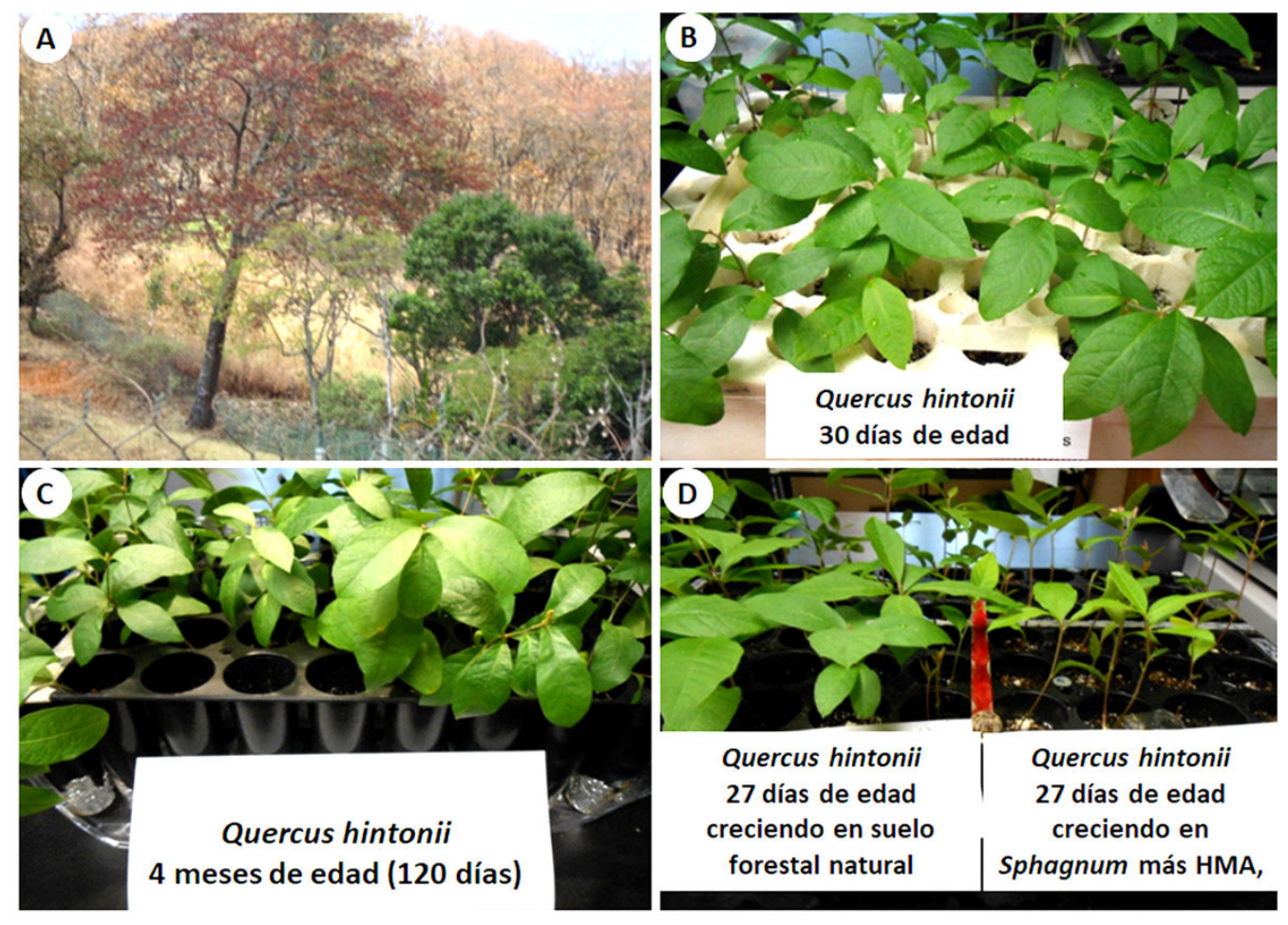

Figura 1. Quercus hintonii. A. Árbol joven en floración con amentos rojos, B. Plantas de Q. hintonii de 30 días de edad creciendo en sustrato de musgo (Sphagnum), e inoculado con HMA, C. Desarrollo del encino a los 120 días de edad, D. un comparativo del crecimiento con suelo forestal natural de su hábitat a los 27 días de edad vs. Sphagnum estéril inoculado con HMA. 
micorrízico arbuscular $(\mathrm{Sph}+\mathrm{I})$, con 11 réplicas cada uno. En ambos casos experimentales, a cada tubete, con excepción de los controles, se le agregó $5 \mathrm{~g}$ de inóculo de hongos micorrízicos arbusculares (HMA), Micofert ${ }^{\circledR}$, que es un biofertilizante no nativo, proveniente de un ambiente tropical, de origen cubano. Este biofertilizante estaba contenido en cascarilla de arroz y turba. Micofert contiene un consorcio de HMA compuesto por: Glomus claroideum Schenck \& Smith, G. etunicatum Becker \& Gerdemann, Kuklospora kentinensis (Wu \& Liu) Oehl \& Sieverd y Diversispora spurca (Pfeiffer, Walker \& Bloss) Walter \& Schuessler, y un total de 107443 esporas $\mathrm{dm}^{-3}$ (Ley-Rivas et al. 2011). El SFN empleado como inóculo nativo en el segundo experimento no se caracterizó en cuanto a su composición de especies de HMA, pero contuvo en promedio 287 esporas $100 \mathrm{~g}^{-1}$ de suelo seco. Las charolas sembradas se regaron periódicamente y mantuvieron en una cámara de crecimiento con $12 \mathrm{~h}$ luz/oscuridad a $25^{\circ} \mathrm{C}$ y al final de cada experimento se extrajeron las plántulas para su evaluación a los 4, 6 y 7 meses de edad en el caso del experimento I, y únicamente a los 4 meses en el caso del experimento II.

Mediciones del desarrollo de las plántulas de encino. En ambos experimentos, a las plántulas de cada unidad experimental se les midió la longitud del tallo y de la raíz con una cinta métrica graduada, así como el diámetro del tallo con un Vernier digital y el número de hojas fue por conteo directo. Estas mediciones se realizaron en las tres fechas durante el experimento I y al final del experimento II. Al final de ambos experimentos se evaluó el peso seco del tallo con las hojas y el de la raíz; así como el peso seco total de las plántulas de $Q$. hintonii, a partir de la cosecha del total de las plántulas y de secarlas en bolsas de papel de estraza en un horno a $40{ }^{\circ} \mathrm{C}$ hasta peso constante.

Evaluación de la colonización micorrízica arbuscular y por endófitos. Las plantas fueron removidas completamente del Sphagnum o del suelo y lavadas con agua manualmente sin dañar la raíz. Las raíces finas poco lignificadas se aclararon con $\mathrm{KOH}$ al $10 \%$ por $48 \mathrm{~h}$ a temperatura ambiente y posteriormente se cubrieron con una solución compuesta de $567 \mathrm{ml}$ de agua más $30 \mathrm{ml}$ de peróxido de hidrógeno al $10 \%$ y $3 \mathrm{ml}$ de hidróxido de amonio durante $20 \mathrm{~min}$, seguido de un lavado con agua destilada. Así, todas las raíces fueron lavadas con $\mathrm{HCl}$ al $10 \%$ por 15 min para neutralizar el $\mathrm{KOH}$ y posteriormente se tiñeron con azul de tripano $(0.08 \%)$ en lactoglicerol (ácido láctico + glicerol + agua) en proporciones 1:1:1 $\mathrm{v} / \mathrm{v} / \mathrm{v}$ (Phillips \& Hayman 1970). Las raíces teñidas se almacenaron en lactoglicerol. Se realizaron preparaciones permanentes de las raíces con alcohol polivinílico en lactoglicerol (PVLG) de cada una de las plántulas. Las preparaciones contenían 20 segmentos de raíces finas de aproximadamente un centímetro de longitud, donde se evaluó el porcentaje de colonización de los hongos a través de la cuantificación de las estructuras características de HMA (hifas cenocíticas, ovillos, arbúsculos, vesículas y esporas) y hongos endófitos (hifas hialinas o melanizadas septadas (MSE), células moniliformes (CME), microesclerocios (MES), like-clamidosporas y otros (Hernández-Cuevas et al. 2008).

Este procedimiento permitió también visualizar las estructuras con un microscopio Olympus BX41 (Tokyo, Japón) con cámara incluida, a través de la cual se obtuvieron las fotografías a $20 x, 40 x$ y $100 x$. Con un microscopio estereoscópico binocular Modelo Leica Z-45L Stereozoom 2000, oculares de 15x, objetivo 1.0x (rango de aumentos a zoom de $10.5 \mathrm{x}$ a $45 \mathrm{x}$ ) con iluminación para luz transmitida y luz incidente (Heerbrugg, Suiza), se hizo la valoración de cada estructura fúngica para calcular los porcentajes de colonización por HMA y HESO/HES en las raíces de las plántulas de $Q$. hintonii creciendo en los diferentes tratamientos experimentales.

Análisis de los datos. Los datos del experimento I se analizaron con un ANOVA de dos factores: (i) tratamiento (Trat) -con y sin inoculante- y (ii) tiempo (T), seguido de una prueba de Tukey. En el caso del experimento II los datos se analizaron con una ANOVA de un factor (tratamiento), seguido de una prueba de Tukey. Previo al análisis y cuando se requirió, los datos fueron transformados a Log10 para cumplir los supuestos de la distribución normal (Sokal \& Rohlf 1995). En el caso del experimento I se realizó un análisis de correlación de Pearson entre las variables de crecimiento y de colonización. Todos los análisis se realizaron en el programa STATISTICA ver 6.0 (Statsoft $2000^{\circledR}$ ) y en todos los casos, los promedios se consideraron diferentes con $p \leq 0.05$.

\section{Resultados}

Las plántulas de $Q$. hintonii se desarrollaron saludablemente en ambos experimentos (Figura 2A-D), sin observarse cambios morfológicos ni síntomas de enfermedad, tanto en las raíces como en la parte aérea. Se registraron estructuras pertenecientes principalmente a dos grupos de hongos colonizando las raíces del encino en estudio. El primer grupo fueron hongos endófitos llamados septados obscuros (HESO), característicos por su color pardo e hifas septadas melanizadas (Figura 2E) y por la formación de microesclerocios melanizados típicos de estos hongos (Figura 2F, G). El segundo grupo de hongos endófitos (HES) registrado se caracteriza por presentar también hifas septadas pero hialinas, que se tiñen con azul de tripano y que se les conocen como hifas "run off", las 
cuales se localizan superficialmente, así como inter e intracelularmente en el tejido cortical de las raíces del encino (Figura 2H-J). Estas hifas se ramifican, formando redes externas e intracelularmente que desarrollan estructuras semejantes a vesículas y microesclerocios que se tiñen de color azul intenso (Figura 2H-M). Estos hongos endofíticos septados predominaron en la colonización de las plántulas de $Q$. hintonii, en particular a los cuatro meses de sembradas las bellotas, que fue cuando ya se encontraban colonizadas las raíces como se observa en las microfotografías (Figura 2).
En el experimento I a pesar de haber inoculado con un consorcio de HMA, no se observó colonización en las raíces por estos hongos, no se apreciaron hifas cenocíticas, arbúsculos, ovillos, vesículas, ni esporas características del phylum Glomeromycota, durante los siete meses de crecimiento de las plántulas del encino. De los HES se observaron células moniliformes (CME) (Figura 2H-M), que son semejantes a las vesículas de la micorriza arbuscular, pero que, dentro de las células corticales, las hifas se modifican vesicularmente y se dividen por medio de septos formando racimos globosos (like-clamidosporas) que también se tiñen intensamente con azul de tripano.
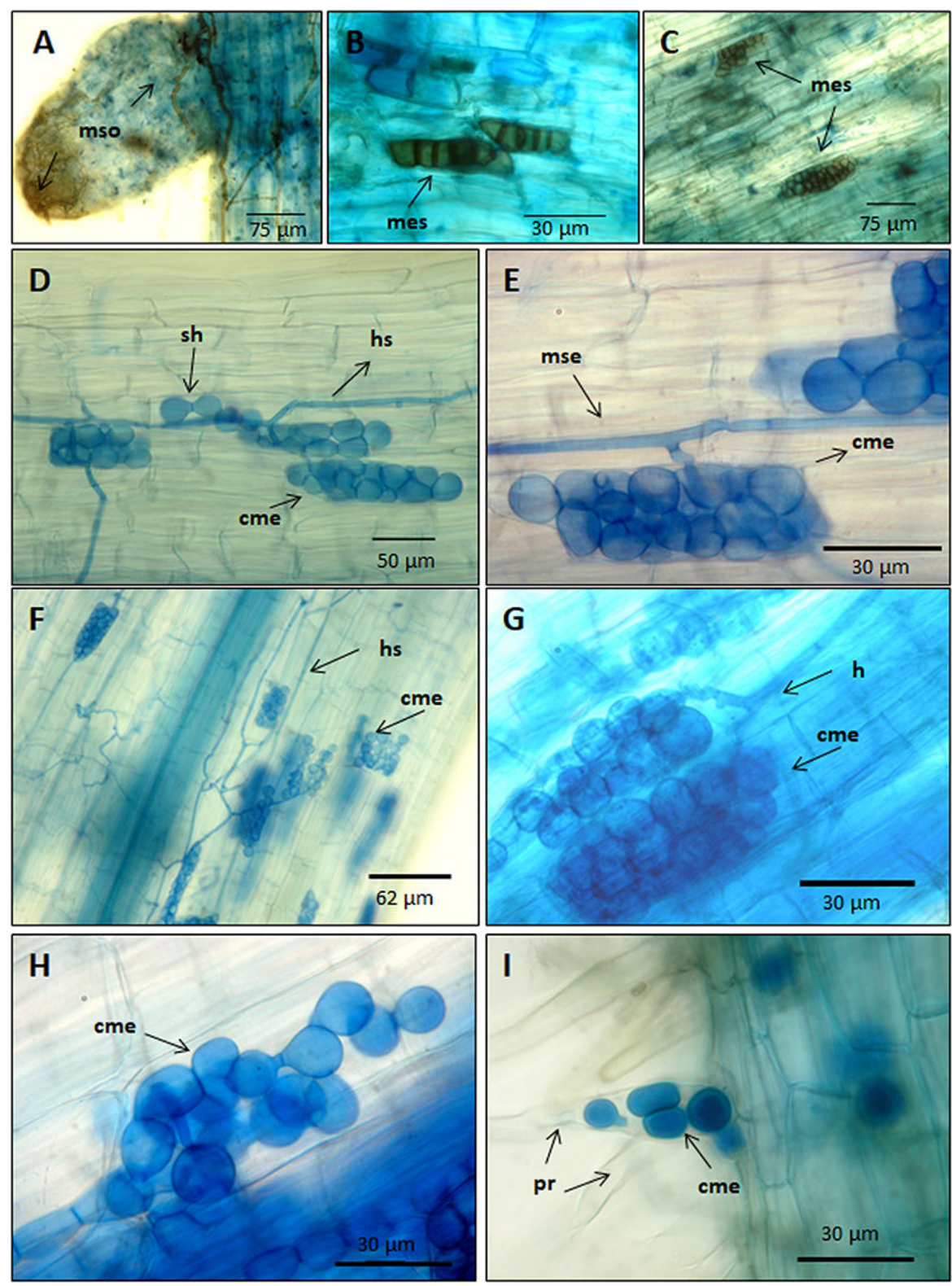

Figura 2. Estructuras de los hongos endofíticos colonizando las raíces de $Q$. hintonii, creciendo en sustrato de Sphagnum e inoculado con hongos micorrízicos arbusculares (HMA Micofert). Leyendas: mso: micelio septado oscuro, mes: microesclerocios, sh: septo de la hifa, hs: hifa septada, cme: células moniliformes, mse: micelio septado, h: hifa, pr: pelo radical. 
En el experimento I también se observó a lo largo del tiempo, un crecimiento positivo tanto en las plántulas inoculadas, como en las no inoculadas (Figuras 3, 4); sin embargo, sólo a los cuatro meses de edad las plántulas inoculadas tuvieron significativamente mayor número de hojas y peso seco de hojas, raíces y biomasa total (Figura 3A-D), así como tallos de mayor diámetro (Figura 4A) que las plántulas control; mientras que la longitud del tallo de las plántulas no fue afectada por la presencia del inóculo (Figura 4B), y la longitud de la raíz fue mayor en las plántulas no inoculadas a los cuatro y seis meses de edad (Figura 4C). Con excepción de la longitud del tallo y de la raíz, todas las demás variables de crecimiento no difirieron significativamente entre los tratamientos cuando las plántulas tuvieron seis y siete meses de edad (Figuras 3, 4). En este mismo experimento, los encinos inoculados de cuatro meses de edad tuvieron mayor colonización por HES que los controles (Figura 5A), con 32, 51 y $7 \%$ de células moniliformes (CME), micelio septado endofítico (MSE) y otras estructuras fúngicas, respectivamente. En contraste, las plántulas de seis y siete meses de edad tuvieron porcentajes más bajos (12-14\%) de colonización por estas estructuras y no fueron diferentes de las plántulas control. Los porcentajes de colonización por CME y MSE correlacionaron negativamente con el diámetro del tallo (CME: $r=-0.38$; MSE: $r=-0.31$; $p \leq 0.05$ ), peso seco de hojas (CME: $r=-0.31$; MSE: $r=-0.24 ; p \leq 0.05$ ), peso seco de raíz (CME: $r=-0.28$; MSE: $r=-0.22 ; p \leq 0.05)$ y biomasa total (CME: $r=-0.37$; MSE: $r=-0.31 ; p \leq 0.05)$ al finalizar los siete meses de crecimiento.

En el experimento II, las plántulas de $Q$. hintonii crecidas en SFN tuvieron significativamente mayor longitud de raíz y biomasa seca total que las crecidas en $\mathrm{SFN}+\mathrm{I}$ y en $\mathrm{Sph}+\mathrm{I}$, tratamientos que tuvieron a su vez un efecto similar sobre estas variables; mientras que las plántulas creciendo en Sph tuvieron los valores más bajos de longitud de raíz y biomasa seca total (Tabla 1). La biomasa seca del tallo y de las hojas fue mayor en plántulas crecidas en SFN, en SFN+I y en Sph +I que en aquellas crecidas en Sph (Tabla 1). En contraste, otras variables de crecimiento no fueron afectadas por estos tratamientos (Tabla 1). La colonización por micelio septado, microesclerocios y otras estructuras fúngicas endofíticas en las raíces de las plántulas de $Q$. hintonii, fue mayor cuando éstas crecieron en SFN y SFN+I, y menor cuando crecieron en $\mathrm{Sph}+\mathrm{I}$ o en Sph (Figura 5B). En contraste, la colonización por células moniliformes no difirió entre tratamientos (Figura 5B). Los valores más bajos de colonización por estructuras endofíticas $(\leq 4 \%)$ se registraron en plántulas control crecidas en Sph.

\section{Discusión}

Los hongos endófitos septados en la raíz, denominados en general por sus características como HESO y HES, pueden cohabitar o no con los HMA sin causar un daño aparente a la planta y en algunos casos promueven el crecimiento de sus hospederos (Jumpponen \& Trape 1998, Jumpponen 2001, Heredia-Acuña et al. 2014, Shankar Naik 2019, Yan et al. 2019, Terhonen et al. 2019). Las estructuras endofíticas, pertenecientes a los dos probables grupos de hongos endófitos septados, detectadas en las raíces de $Q$. hintonii en ambos experimentos, evidencian una importante interacción de esta especie de encino con HESO y HES. Se considera que algunas de estas estructuras, como las vesículas y los microesclerocios, pueden almacenar substancias de reserva para la germinación y/o ser estructuras de propagación y resistencia ante condiciones ambientales adversas (Jumpponen 2001). Además, se menciona que fisiológicamente los microesclerocios podrían participar en el intercambio nutrimental entre el hongo y la planta, pero se desconoce si todos estos hongos forman microesclerocios y si la reducida superficie de contacto y condición de melanización de esta estructura pudieran limitar el intercambio de nutrimentos (HerediaAcuña et al. 2014). Asimismo, las hifas septadas hialinas, las células moniliformes y aquellas estructuras similares a clamidosporas intracelulares observadas en las raíces de este encino son parecidas a las reportadas para estos hongos en otras plantas (Zubek et al. 2011, Muthukumar et al. 2014), sugiriendo que además de los HESO existen también otros HES colonizando las raíces de esta especie de encino.

El presente estudio también muestra que los hongos endófitos registrados en las raíces de $Q$. hintonii no cohabitaron con los HMA y tampoco causaron daño alguno a las plántulas. Varios estudios indican que los encinos favorecen la presencia de casi todos los phyla fúngicos (Zubek et al. 2011, Toju et al. 2013, Kwaśna et al. 2016), aunque predominan los Ascomycota y Basidiomycota (Southworth 2013, Chávez-Vergara et al. 2016). Así, los resultados sugieren que la presencia de los HESO/HES en las raíces de $Q$. hintonii podría ser atribuida a la prevalencia del phylum Ascomycota, debido a que las especies de HESO reportadas con mayor frecuencia en la literatura pertenecen a este phylum y a los órdenes Helotiales, Magnaporthales, Chaetosphaeriales y Capnodiales (Heredia-Acuña et al. 2014, Terhonen et al. 2019); aunque es aún necesario emplear a futuro metodologías más sensibles que permitan explorar con mayor precisión la composición taxonómica de estos hongos. En contraste, si bien se reporta la presencia de HMA en el suelo de los bosques de encino (Daniels \&Trappe 1979, Olivera-Morales et al. 2011, González-Cortés et al. 2012, Varela-Fregoso et al. 2017) y que en varias especies de Quercus las raíces pueden ser simultáneamente colonizadas por hongos micorrízicos y otros endofíticos (Valentine et al. 2002, Dickie et al. 2001, Bainard et al. 2011, Zubek et al. 2011, Toju et al. 2013, Terhonen et al. 2019), la ausencia de estructuras características de los HMA en las plántulas 

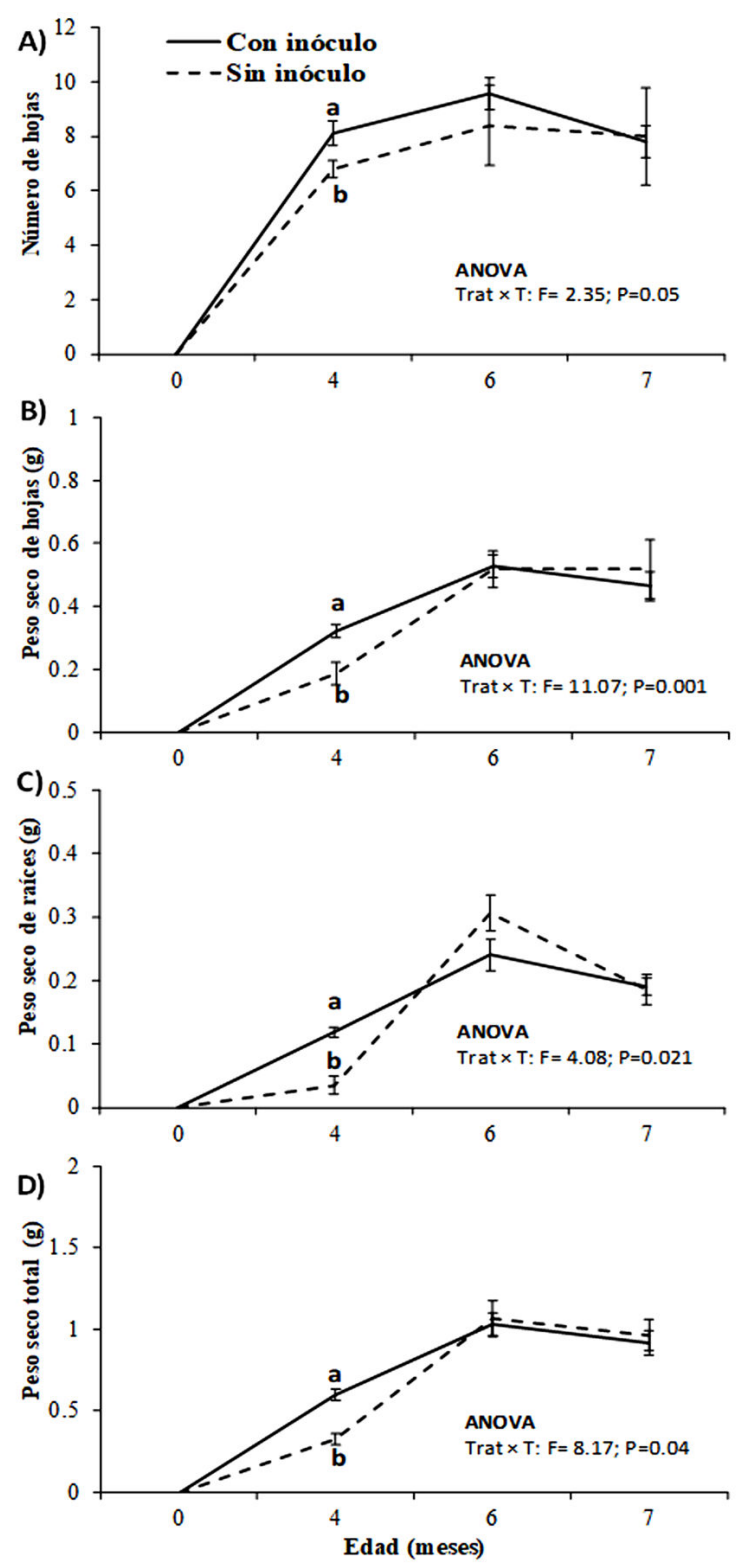

Figura 3. Plántulas de Quercus hintonii creciendo en sustrato Sphagnum con y sin inóculo micorrízico (Micofert) dentro de una cámara de crecimiento. Se muestran promedios ( \pm error estándar): A. Número de hojas, B. Peso seco de la hoja, C. Peso seco del tallo, y D. Biomasa seca total. El efecto significativo de los tratamientos (Trat) y del tiempo ( $\mathrm{T}$, edad de la plántula) están indicados sobre cada gráfico con los valores del $F$-ANOVA. Letras distintas indican diferencias significativas entre tratamientos dentro del mismo tiempo con Tukey $(p \leq 0.05)$.
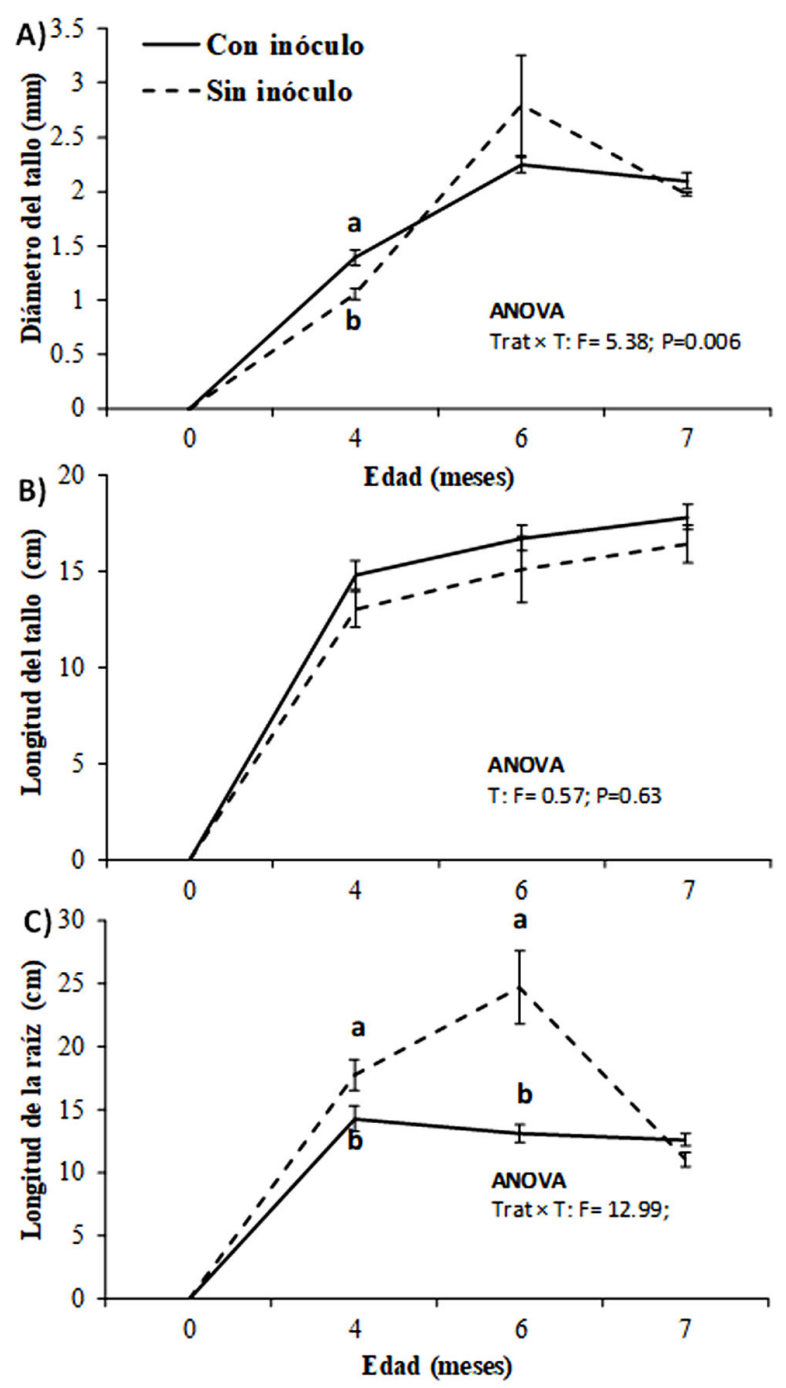

Figura 4. Plántulas de Quercus hintonii creciendo en sustrato Sphagnum con y sin inóculo micorrízico (Micofert) dentro de una cámara de crecimiento. Se muestran promedios ( \pm error estándar) de: A. Diámetro de tallo, B. Longitud del tallo, y C. Longitud de raíz. Los efectos significativos de los tratamientos (Trat) y del tiempo ( $\mathrm{T}$, edad de la plántula) están expresados sobre cada gráfico con los valores del F-ANOVA. Células moniliformes endofíticas (CME), Micelio de endófitos septados (MES). Inóculo micorrízico arbuscular no nativo. Letras distintas indican diferencias significativas entre tratamientos dentro del mismo tiempo con Tukey $(p \leq 0.05)$. 
a) EXPERIMENTOI

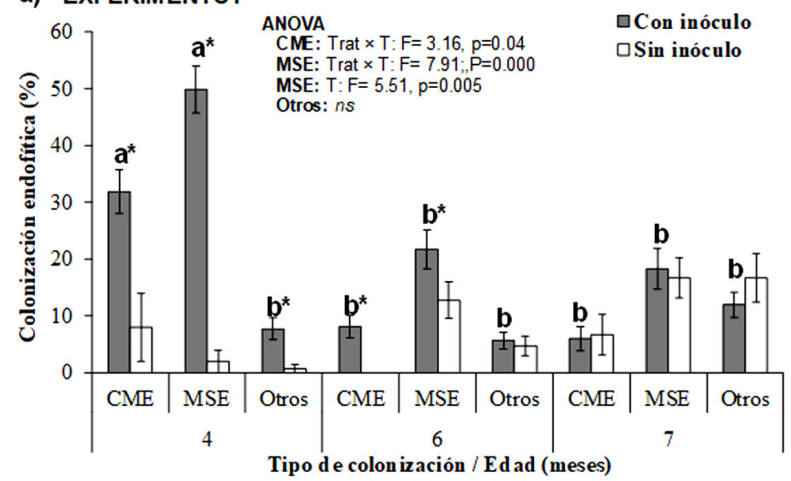

b) EXPERIMENTOII

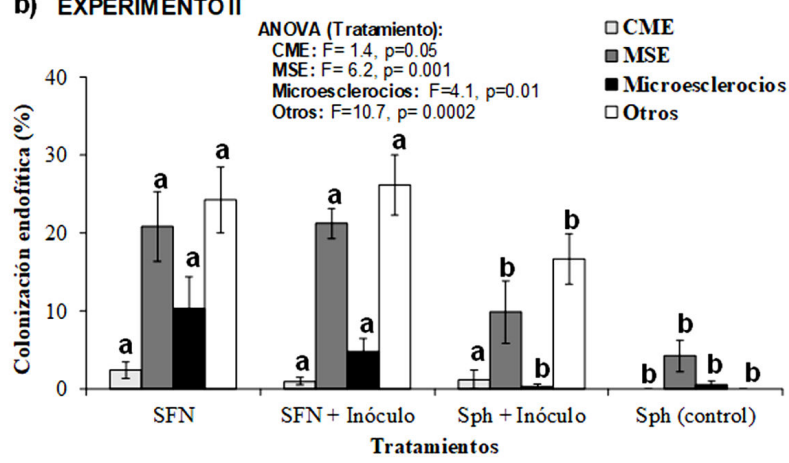

Figura 5. Porcentajes de colonización por hongos endofíticos septados en las raíces de plántulas de Quercus hintonii crecidas en una cámara de crecimiento. Se muestran promedios $( \pm$ error estándar) de: A. Colonización por estructuras fúngicas en tres diferentes tiempos del crecimiento de las plántulas (Experimento I), y B. Variación de la colonización de estructuras fúngicas entre los tratamientos de inoculación (Experimento II). Los efectos significativos de los tratamientos (Trat) y/o el tiempo ( $\mathrm{T}$, edad de las plántulas) están expresados sobre cada gráfico con los valores del F-ANOVA. Células moniliformes endofíticas (CME), Micelio de endófitos septados (MES), suelo forestal natural (SFN), $\mathrm{Sph}=$ Sphagnum. Inóculo micorrízico arbuscular no nativo. Con una prueba de Tukey $(p \leq 0.05)$, los asteriscos indican diferencias significativas entre tratamientos dentro de un mismo tiempo, y letras distintas indican diferencias significativas entre meses para una misma estructura fúngica colonizando las raíces.

crecidas tanto con el inóculo micorrízico (experimento I), como en el suelo forestal natural con o sin él inóculo micorrízico (experimento II), indica al menos tres posibilidades: i) el inoculante Micofert no fue efectivo en esta especie de encino debido a su origen tropical o a la cantidad y composición de especies de HMA (Ley-Rivas et al. 2011); ii) $Q$. hintonii no se asocia, al menos bajo las condiciones analizadas, con los HMA como ocurre en otras plantas de bosques templados (Read \& Pérez-Moreno 2003, Varela-Fregoso et al. 2017), ya que la variación en los nutrientes a lo largo del experimento pudo haberse reflejado en cierta independencia micorrízica por parte del encino; y iii) esta especie de encino podría no depender de estos simbiontes para su desarrollo, dado que los dos experimentos de este estudio muestran que sus raíces no fueron colonizadas por los HMA del inóculo (no nativo) ni del suelo forestal natural. No obstante, es aún necesario evaluar cómo las propiedades del suelo podrían determinar la composición de HMA y en consecuencia mediar el establecimiento o no de la asociación micorrízica en este encino, debido que se ha demostrado en otros bosques de encino, que los hongos del suelo son espacial y temporalmente dinámicos en abundancia, composición, biomasa y actividad (Saikkonen 2007, Voříšková et al. 2014, Chávez-Vergara et al. 2016, Kwaśna et al. 2016, Toju et al. 2013, Terhonen et al. 2019).

A pesar de la ausencia de HMA en las raíces de Q. hintonii, las plántulas de cuatro meses de edad en el experimento I, fueron favorecidas en su desarrollo por la presencia del inóculo micorrízico, sugiriendo que los hongos endófitos septados, provenientes del sustrato en que venía el inóculo no nativo (cascarilla de arroz y turba), al colonizar la raíz, promovieron el crecimiento y la ganancia de biomasa seca en las plántulas de este encino. Al respecto, se ha documentado que los HESO y HES, al inhibir el crecimiento de patógenos y mejorar la tolerancia al estrés edáfico, pueden acelerar el crecimiento, mejorar la nutrición e incrementar la biomasa de brotes y raíces de sus hospederos (Arnold \& Herre 2003, Herrera et al. 2007, Rodriguez \& Redman 2008, Rodriguez et al. 2009, Newsham 2011, Shankar Naik 2019, Yan et al. 2019). Esto en conjunto, sugiere que los hongos endófitos septados podrían tener un papel funcional crítico y semejante al de los HMA para el desarrollo de $Q$. hintonii, al menos durante los primeros cuatro meses de edad, como se ha reportado en otras especies de plantas, en diferentes ecosistemas a nivel mundial (Jumpponen 2001, Newsham et al. 2009, PorrasAlfaro \& Bayman 2011, Heredia-Acuña et al. 2014). Asimismo, en las etapas posteriores a los cuatro meses de edad, el desarrollo de las plántulas de $Q$, hintonii pudiera depender menos de los hongos endófitos septados, toda vez que se ha observado que en otras especies de encino (e.g. Q. rugosa), durante los primeros meses de crecimiento, la micorriza arbuscular es más importante y posteriormente es desplazada por la ectomicorriza (Lodge \& Wentworth 1990, Olivera-Morales et al. 2011). Esto está apoyado por las correlaciones negativas entre la colonización por estructuras endofíticas de los HESO/HES y los parámetros de crecimiento de las plántulas de $Q$. hintonii (experimento I), que sugieren que a mayor edad este encino podría ser menos dependiente de estos hongos para su desarrollo. No obstante, el hecho de que los hongos endofíticos, como parte de los microbiomas de los bosques, puedan funcionalmente mejorar la adecuación no solo de los árboles 
Tabla 1. Experimento II: Efecto de cuatro diferentes tratamientos de inoculación sobre el desarrollo promedio ( \pm error estándar) de las plántulas de Quercus hintonii después de cuatro meses de crecimiento bajo condiciones controladas.

\begin{tabular}{lllllll}
\hline & \multicolumn{3}{c}{ Tratamientos } & \multicolumn{3}{c}{ ANOVA } \\
\cline { 2 - 7 } & SFN & SFN + I & Sph + I & Sph & F & $\boldsymbol{p}$ \\
\hline Número de hojas & $7.7(0.2)^{\mathrm{a}}$ & $7.7(0.2)^{\mathrm{a}}$ & $8.0(0.2)^{\mathrm{a}}$ & $8.2(0.1)^{\mathrm{a}}$ & 0.2 & 0.88 \\
Longitud del tallo (cm) & $15.1(1.0)^{\mathrm{a}}$ & $14.8(0.9)^{\mathrm{a}}$ & $14.2(0.7)^{\mathrm{a}}$ & $13.55(0.7)^{\mathrm{a}}$ & 0.5 & 0.64 \\
Diámetro del tallo (cm) & $1.63(0.09)^{\mathrm{a}}$ & $1.57(0.09)^{\mathrm{a}}$ & $1.59(0.06)^{\mathrm{a}}$ & $1.59(0.08)^{\mathrm{a}}$ & 0.08 & 0.96 \\
Longitud de la raíz (cm) & $13.3(0.8)^{\mathrm{a}}$ & $11.9(0.4)^{\mathrm{b}}$ & $12.4(0.4)^{\mathrm{b}}$ & $10.1(0.4)^{\mathrm{c}}$ & $\mathbf{2 . 4}$ & $\mathbf{0 . 0 5}$ \\
Peso seco de las hojas (g) & $0.26(0.02)^{\mathrm{a}}$ & $0.21(0.02)^{\mathrm{a}}$ & $0.22(0.02)^{\mathrm{a}}$ & $0.19(0.02)^{\mathrm{a}}$ & 1.0 & 0.38 \\
Peso seco de tallo y hojas $(\mathbf{g})$ & $0.38(0.03)^{\mathrm{a}}$ & $0.32(0.02)^{\mathrm{a}}$ & $0.32(0.04)^{\mathrm{a}}$ & $0.26(0.03)^{\mathrm{b}}$ & $\mathbf{1 . 9}$ & $\mathbf{0 . 0 4}$ \\
Peso seco de la raíz (g) & $0.09(0.01)^{\mathrm{a}}$ & $0.08(0.01)^{\mathrm{a}}$ & $0.09(0.01)^{\mathrm{a}}$ & $0.08(0.01)^{\mathrm{a}}$ & 0.2 & 0.86 \\
Biomasa seca total $(\mathbf{g})$ & $0.52(0.04)^{\mathrm{a}}$ & $0.41(0.03)^{\mathrm{b}}$ & $0.41(0.05)^{\mathrm{b}}$ & $0.24(0.04)^{\mathrm{c}}$ & 5.8 & $\mathbf{0 . 0 4}$ \\
\hline
\end{tabular}

Los números en negritas corresponden a $p \leq 0.05$ y muestran efectos significativos de algún tratamiento. Letras distintas indican diferencias significativas con la prueba de Tukey $(p \leq 0.05)$. SFN $=$ Suelo forestal natural, $\mathrm{SFN}+\mathrm{I}=$ Suelo forestal natural más inóculo micorrízico, Sph + $\mathrm{I}$ = Sphagnum más inóculo micorrízico, $\mathrm{Sph}=$ Sphagnum $. \mathrm{I}=$ Inóculo micorrízico arbuscular no nativo (Micofert).

individuales, sino también de todo el ecosistema forestal (Terhonen et al. 2019); sugiere que, los HESO y HES asociados a $Q$. hintonii podrían ser críticos para mantener la salud del bosque templado en esta región de México.

El mayor desarrollo de $Q$. hintonii registrado a los cuatro meses de edad, en términos de crecimiento de raíces y ganancia de biomasa seca de tallo, hojas y masa total, fue probablemente, debido a la colonización por HESO y HES provenientes del suelo forestal natural, y menos al efecto de los hongos endófitos septados en el inóculo micorrízico (experimento II); lo que indica que los endófitos septados nativos podrían ser más eficientes como promotores del crecimiento de este encino al inicio de su desarrollo. Lo anterior, se podría relacionar con la adaptación a las condiciones ambientales en que ambos organismos crecen y a la relevancia de los inóculos endofíticos autóctonos sobre los alóctonos, como se ha indicado para los inóculos micorrízicos en otras plantas (Trejo et al. 2011). La nula eficiencia del inóculo alóctono (Micofert) para colonizar las raíces de $Q$. hintonii y la baja eficiencia de los hongos endófitos alóctonos para promover el crecimiento de las plántulas, podría atribuirse a que el inóculo proviene de ambientes tropicales, en contraste con el suelo forestal natural obtenido del bosque templado de donde además Q. hintonii es endémico.

En conclusión, el inoculante con HMA, exitoso en ambientes tropicales, no interaccionó con $Q$. hintonii procedente de un clima templado; pero en forma indirecta se tuvo información valiosa de la colonización por hongos endófitos septados, los cuales no causaron ningún daño a las plántulas, sino que, por el contrario, favorecieron el desarrollo de $Q$. hintonii en sus primeros cuatro meses de vida, lo que podría contribuir a su establecimiento en campo. Los dos experimentos, en conjunto, sugieren que esta especie de encino podría depender para su desarrollo temprano de los hongos endófitos septados (HESO/HES) y no de los HMA, ya que se demostró que sus raíces no fueron colonizadas ni por los HMA del inóculo no nativo ni del suelo forestal natural. Asimismo, este estudio permite hipotetizar para futuras investigaciones, que los hongos endófitos septados nativos podrían ser mejores promotores del crecimiento de las plántulas de $Q$. hintonii durante sus primeras etapas de vida que los endofíticos septados alóctonos, debido a su probable adaptación a las condiciones ambientales prevalecientes en estos bosques. Además, debido a su importancia en el desarrollo de Q. hintonii es necesario, a futuro, caracterizar la comunidad de hongos endofíticos septados habitando el suelo rizosférico de estos bosques.

\section{Agradecimientos}

A Eduardo Chimal Sánchez por el diseño y elaboración de las láminas fotográficas. A dos revisores anónimos cuyos comentarios y sugerencias enriquecieron este manuscrito.

\section{Literatura citada}

Arnold AE, Herre EA. 2003. Canopy cover and leaf age affect colonization by tropical fungal endophytes: ecological pattern and process in Theobroma cacao (Malvaceae). Mycologia 95: 388-398. DOI: https:// doi.org/10.1080/15572536.2004.11833083

Bainard LD, Klironomos JN, Gordon AM. 2011.The mycorrhizal status and colonization of 26 tree species growing in urban and rural environments. Mycorrhiza 
21: 91-96. DOI: https://doi.org/10.1007/s00572-010-03 $\underline{14-6}$

Bonner FT, Vozzo JA. 1987. Seed biology and technology of Quercus. United States of America. US, New Orleans: Department of Agricultural, Forest Service. Genetic and Technology Reproduction. SO-66. DOI: https://doi.org/ $\underline{10.2737 / \text { SO-GTR-66 }}$

Cavender-Bares J. 2018. Diversification, adaptation, and community assembly of the American oaks (Quercus), a model clade for integrating ecology and evolution. New Phytologist 221: 689-692. DOI: https://doi.org/10.1111/ nph. 15450

Chávez-Vergara B, Rosales-Castillo A, Merino A, VázquezMarrufo G, Oyama K, García-Oliva F. 2016. Quercus species control nutrients dynamics by determining the composition and activity of the forest floor fungal community. Soil Biology and Biochemistry 98: 186-195. DOI: https://doi.org/10.1016/j.soilbio.2016.04.015

Daniels AB, Trappe MJ. 1979. Glomus epigaeus sp. nov., a useful fungus for vesicular-arbuscular mycorrhizal research. Canadian Journal of Botany 57: 539-542. DOI: https://doi.org/10.1139/b79-070

Díaz-Pontones D, Reyes-Jaramillo I. 2009. Producción y almacenamiento de bellotas de Quercus hintonii Warburg (Fagaceae) de la Depresión del Balsas, México. Polibotánica 27: 131-143.

Dickie IA, Koide RT, Fayish AC. 2001.Vesicular-arbuscular mycorrhizal infection of Quercus rubra seedlings. New Phytologist 151: 257-264. DOI: https://doi.org/10.1046/ j.1469-8137.2001.00148.x

González-Cortés JC, Vega-Fraga M, Varela-Fregoso L, Martínez-Trujillo M, Carreón-Abud Y, Gavito ME. 2012. Arbuscular mycorrhizal fungal (AMF) communities and land use change: the conversion of temperate forests to avocado plantations and maize fields in central Mexico. Fungal Ecology 5: 16-23. DOI: https://doi.org/10.1016/ j.funeco.2011.09.002

Heredia-Acuña C, Alarcón A, Hernández-Cuevas LV, Ferrera-Cerrato R, Almaraz-Suárez JJ. 2014. Diversidad, ecología e importancia potencial de los hongos endófitos septados obscuros en México. Botanical Sciences 92: 321-333. DOI: https://doi.org/10.17129/botsci.114

Hernández-Cuevas LV, Guadarrama-Chávez P, SánchezGallen I, Ramos-Zapata J. 2008. Micorriza arbuscular, colonización intraradical y extracción de esporas del suelo. In: Álvarez FJ, Monroy-Ata A, eds. Técnicas de Estudio de las Asociaciones Micorrizicas y sus Implicaciones en la Restauración. México, DF: Facultad de Ciencias, Universidad Nacional Autónoma de México, pp.1-27. ISBN: 978-970-32-5527-6

Herrera EA, Mejía LC, Kyllo DA, Rojas E, Maynard Z, Butler A, Van Bael SA. 2007. Ecological implications of anti-pathogen effects of tropical fungal endophytes and mycorrhizae. Ecology 88: 550-558. DOI: https://doi.org/ $\underline{10.1890 / 05-1606}$

INEGI [Instituto Nacional de Estadística y Geografía]. 2009. Prontuario de información geográfica municipal de los Estados Unidos Mexicanos. Tejupilco. México, DF: Clave geoestadística 15082. http://www3.inegi.org.mx/ contenidos/app/mexicocifras/datos_geograficos/15/1508 2.pdf (consultado enero 12, 2018).

Jumpponen A. 2001. Dark septate endophytes -are they mycorrhizal? Mycorrhiza 11: 207-211. DOI: https:// doi.org/10.1007/s005720100112

Jumpponen A, Trape JM. 1998. Dark septate root endophytes: a review of facultative biotrphic rootcolonizing fungi. New Phytologist 140: 295-310. DOI: https://doi.org/10.1046/j.1469-8137.1998.00265.x

Kwaśna H, Szewczyk W, Behnke-Borowczyk J. 2016. Fungal root endophytes of Quercus robur subjected to flooding. Forest Pathology 46: 35-46. DOI: https:// doi.org/10.1111/efp.12212

Ley-Rivas JF, Aliaga L, Moron C, Furrazola E. 2011. Efecto del biofertilizante MICOFERT en la producción de dos variedades de lechuga en Perú. Acta Botánica Cubana 213: 36-39.

Lodge DJ, Wentworth TR. 1990. Negative associations among VA-mycorrhizal fungi and some ectomycorrhizal fungi inhabiting the same root system. Oikos 57: 347-356. DOI: https://doi.org/10.2307/3565964

Muthukumar T, Sathiyaraj G, Priyadharsini P, Uma E, Sathiyadash K. 2014. Arbuscular mycorrhizal and dark septate endophyte fungal associations in ferns and lycophytes of Palni Hills, Western Ghats, southern India. Brazilian Journal of Botany 37: 561-581. DOI: https:// doi.org/10.1007/s40415-014-0085-y

Newsham KK. 2011. A meta-analysis of plant responses to dark septate root endophytes. New Phytologist 190: 783-793. DOI: https://doi.org/10.1111/j.1469-8137.20 $\underline{10.03611 . \mathrm{x}}$

Newsham KK, Upson R, Read DJ. 2009. Mycorrhizas and dark septate root endophytes in polar regions. Fungal Ecology 2: 10-20. DOI: https://doi.org/10.1016/j.fun eco.2008.10.005

Olivera-Morales D, Castillo-Argüero S, Guadarrama $\mathrm{P}$, Ramos-Zapata J, Álvarez-Sánchez J, Hernández-Cuevas L. 2011. Establecimiento de plántulas de Quercus rugosa Née inoculadas con hongos micorrizógenos arbusculares en un bosque templado de México. Boletín de la Sociedad Botánica de México 89: 115-121. DOI: https:// doi.org/10.17129/botsci.372

Phillips JB, Hayman DS. 1970. Improved procedures for clearing roots and staining parasitic and vesiculararbuscular mycorrhizal fungi for rapid assessment of infection. Transactions of the British Mycological Society 55: 158-160. DOI: https://doi.org/10.1016/ $\underline{\mathrm{S} 0007-1536(70) 80110-3}$ 
Porras-Alfaro A, Bayman P. 2011. Hidden fungi, emergent properties: Endophytes and microbiomes. Annual Review Phytopathology 49: 291-315. DOI: https://doi.org/10.114 6/annurev-phyto-080508-081831

Read JD, Pérez-Moreno J. 2003. Mycorrhizas and nutrient cycling in ecosystems - a journey towards relevance? New Phytologist 157: 475-492. DOI: https://doi.org/10. 1046/j.1469-8137.2003.00704.x

Reyes-Jaramillo I. 2006. Quercus hintonii Warb: especie endémica del encinar del SW del Estado de México. Contactos 60: 64-72.

Rodriguez RJ, White JF, Arnold, AE, Redman RS. 2009. Fungal endophytes: diversity and functional roles. New Phytologist 18: 314-330. DOI: https://doi.org/10.1111/ j.1469-8137.2009.02773.x

Rodriguez RJ, Redman R. 2008. More than 400 million years of evolution and some plants still can't make it on their own: plant stress tolerance via fungal symbiosis. Journal of Experimental Botany 59: 1109-1114. DOI: https://doi.org/10.1093/jxb/erm342

Romero-Rangel S, Rojas-Zenteno EC, Rubio-Licona LE. 2015. Encinos de México (Quercus, Fagaceae). México, Ciudad de México: Universidad Nacional Autónoma de México-Facultad de Estudios Superiores Iztacala. ISBN: 978-607-02-6650-8

Rzedowski J. 2006. La Vegetación de México. México, Ciudad de México: 1ra. Edición digital, Comisión Nacional para el Conocimiento y Uso de la Biodiversidad.

Saikkonen K. 2007. Forest structure and fungal endophytes. Fungal Biology Reviews 21: 67-74. DOI: https://doi.org/ $\underline{\text { 10.1016/j.fbr.2007.05.001 }}$

Shankar Naik S. 2019. Functional roles of fungal endophytes in host fitness during stress conditions. Symbiosis 79: 99-115. DOI: https://doi.org/10.1007/ s13199-019-00635-1

Sokal R, Rohlf F. 1995. Biometry. USA, San Francisco: Freeman and Company. ISBN: 84-291-1862-4

Southworth D. 2013. Oaks and mycorrhizal fungi. In: Chuteira CA, Grao AB, eds. Oak ecology, types and management. New York, NY: Nova Science Publishers. pp. 207-218. ISBN: 978-1-61942-493-7

StatSoft 2000. Statistica ver. 6.0. for Windows [Computer program manual]. Tulsa, OK.

Terhonen E, Blumenstein K, Kovalchuk A, Asiegbu FO. 2019. Forest tree microbiomes and associated fungal endophytes functional roles and impact on forest health. Forest 10: 1-33 DOI: https://doi.org/10.3390/f10010042

Toju H, Yamamoto S, Sato H, Tanable AS, Gilbert GS, Kadowaki K. 2013. Community composition of root associated fungi in a Quercus dominated temperate forest: "codominance" of mycorrhizal and root endophytic fungi. Ecology and Evolution 3: 1281-1293. DOI: https://doi.org/10.1002/ece3.546

Trejo D, Ferrera-Cerrato R, García R, Varela L, Lara L, Alarcón A. 2011. Efectividad de siete consorcios nativos de hogos micorrízicos arbusculares en plantas de café en condiciones de invernadero y campo. Revista Chilena de Historia Natural 84: 23-31. DOI: http://dx.doi.org/ $\underline{\text { 10.4067/S0716-078X2011000100002 }}$

Valencia-A S. 2004. Diversidad del género Quercus (Fagaceae) en México. Boletín de la Sociedad Botánica de México 75: 33-53. DOI: http://dx.doi.org/10.17129/ botsci. 1692

Valentine LL, Fiedler TL, Haney SR, Berninghausen HK, Southworth D. 2002. Biodiversity of mycorrhizas on Garry oak (Quercus garryana) in a southern Oregon savanna. USA, San Diego: Forest Service General Technical Report. PSW-GTR-184, pp. 151-157.

Varela-Fregoso L, Mora-Velázquez A, Chávez-Hernández CG, Martínez-Bernal A, García-Sánchez R, ChimalSánchez E, Montaño NM. 2017. Acaulospora alpina y Ambispora fennica, dos registros nuevos de hongos micorrizógenos arbusculares para México. Revista Mexicana de Biodiversidad 88: 496-501. DOI: https:// doi.org/10.1016/j.rmb.2017.06.005.

Voříšková J, Brabcová V, Cajthaml T, Baldrian P. 2014. Seasonal dynamics of fungal communities in a temperate oak forest soil. New Phytologist 201: 269-278 DOI: https://doi.org/10.1111/nph.12481

Yan L, Zhu J, Zhao X, Shi J, Jiang Ch, Shao D. 2019. Beneficial effects of endophytic fungi colonization on plants. Applied Microbiology and Biotechnology 103: 3327-3340. DOI: https://doi.org/10.1007/s00253-019-09 713-2

Zubek S, Nobis M, Błaszkowski J, Mleczko P, Nowak A. 2011. Fungal root endophyte associations of plants endemic to the Pamir Alay Mountains of Central Asia. Symbiosis 54: 139-149. DOI: https://doi.org/10.1007/ $\underline{\mathrm{s} 13199-011-0137-\mathrm{Z}}$
Editor de sección: Pablo Cuevas Reyes

Contribución de los autores: IR-J, realizó el trabajo de campo, diseñó los experimentos, recopiló los datos y escribió el manuscrito. NMM, revisó y analizó los datos y escribió el manuscrito. ESG, aplicó los tratamientos experimentales, monitoreó y obtuvo los datos de ambos experimentos. 